\title{
LncRNA NEAT1/microRNA-129-5p/ SOCS2 axis regulates liver fibrosis in alcoholic steatohepatitis
}

Junfeng Ye ${ }^{1}$, Yuanqiang Lin², Ying Yư and Di Sun ${ }^{3^{*}}$

\begin{abstract}
Background: Long non-coding RNA nuclear paraspeckle assembly transcript 1 (NEAT1) has been reported to play an essential role in non-alcoholic fatty liver disease. However, the role of NEAT1 in regulation of alcoholic steatohepatitis (ASH) remains largely unknown. This study aims to explore the role of NEAT1 in ASH by mediating microRNA-129-5p (miR-129-5p) targeting suppressor of cytokine signaling 2 (SOCS2).

Methods: NEAT1, miR-129-5p and SOCS2 expression in serum of ASH patients were assessed. In the in vitro cellular experiment, we transfected siRNAs, oligonucleotides or plasmids into ethanol-induced AML-12 mouse hepatocytes to alter NEAT1 and miR-129-5p expression, and inflammatory factors and lipid content were determined. In the in vivo animal experiment, we injected lentiviruses carrying siRNAs, oligonucleotides or plasmids onto ASH mice (ASH induced by feeding mice a Lieber-DeCarli ethanol diet) to alter NEAT1 and miR-129-5p expression through the tail vein. Serum liver function, blood lipids and inflammatory factors were detected; liver histopathology, liver cell apoptosis, and fibrosis were observed. The relationship between NEAT1 and miR-129-5p, or between miR-129-5p and SOCS2 was verified.
\end{abstract}

Results: MiR-129-5p was reduced while NEAT1 and SOCS2 were elevated in ASH. Inhibited NEAT1 or elevated miR129-5 $p$ suppressed the elevated lipid metabolism and restrained inflammation reaction in ethanol-stimulated AML-12 cells. The promoted miR-129-5p and inhibited NEAT1 could improve the liver function and repress blood lipid, inflammation reaction, hepatocyte apoptosis and liver fibrosis in ethanol-induced ASH mice. Furthermore, NEAT1 could negatively regulate miR-129-5p to target SOCS2.

Conclusion: We have found that the inhibited NEAT1 could suppress liver fibrosis in ASH mice by promoting miR$129-5 p$ and restraining SOCS2, thereby decelerating the development of ASH.

Keywords: Alcoholic steatohepatitis, Long non-coding RNA nuclear paraspeckle assembly transcript 1, MicroRNA129-5p, Suppressor of cytokine signaling 2, Liver fibrosis

\section{Background}

As the largest solid organ, liver serves as a pivotal role in metabolism and detoxication in human body. Liver injury is the main cause that greatly contributes to the

*Correspondence: Sundi2332@163.com

${ }^{3}$ Department of Colorectal \& Anal Surgery, First Hospital, Jilin University, No. 71 Xinmin street, Changchun 130021, Jilin, People's Republic of China Full list of author information is available at the end of the article risk of human health globally, especially in Asian countries [1]. Second to viral hepatitis, alcoholic liver disease (ALD) and non-alcoholic fatty liver disease (NAFLD) are the commonest liver diseases in developed countries [2]. ALD is a deleterious result of excessive alcohol consumption, resulting in a great burden and a loss of socioeconomic productivity in the world [3]. Alcohol consumption would promote lipogenesis and mobilization of lipids and decelerate lipid catabolism, causing a 
lipid accumulation in hepatocytes, called fatty liver. In some cases, alcohol consumption results in an inflammatory reaction, which is known as alcoholic steatohepatitis (ASH) if there is hepatic lipid deposition [4]. Although ASH progresses slowly, the continual chronic liver injury and inflammation would finally lead to progressive fibrosis and cirrhosis, then may promote the progression of hepatocellular carcinoma (HCC) [4]. As an acute inflammatory liver disease associated with poor prognosis [5], novel and efficient treatment for ASH is urgently needed.

Long noncoding RNAs (lncRNAs) are a kind of noncoding RNAs of over 200 nucleotides and have little or no protein-coding ability [6]. Abnormal expression of particular lncRNAs has been demonstrated to be related with development of human diseases. LncRNA nuclear paraspeckle assembly transcript 1 (NEAT1) is a novel lncRNA that transcribed from many endocrine neoplasia locus [7]. As reported, NEAT1 promoted inflammatory response in sepsis-induced liver injury [8], and NEAT1 also facilitated hepatic lipid accumulation and exacerbated NAFLD $[9,10]$. Furthermore, microRNAs (miRNAs) regulate expression of target gene through binding to the $3^{\prime}$-untranslated region ( $\left.3^{\prime} \mathrm{UTR}\right)$ of target gene mRNA, causing degradation or translational suppression of mRNA [11]. Multiple miRNAs have been identified in ASH, including miR-31, miR-191 and miR-451 [12]. As a member of miRNAs, miR-129-5p has been identified to regulate the progression of HCC [13, 14]. In addition, the regulatory relation between NEAT1 and miR-129-5p has been revealed in HCC [15] and hepatoblastoma [16], while this relation has not been unraveled in ASH. Additionally, suppressor of cytokine signaling (SOCS) family was initially recognized as suppressors of the cytokine signaling [17]. As one of the SOCS family, SOCS2 was clarified as a feedback suppressor of the growth hormone/insulin-like growth factor axis [18]. It has been clarified that SOCS2 participated in NAFLD [19] and hepatic steatosis [20]. Nevertheless, the impact of SOCS2 on ASH, as well as the target relation between SOCS2 and miR-129-5p remains to be further explored. This study was designed to elucidate the role of the NEAT1/ miR-129-5p/SOCS2 axis in the progression of ASH, and we inferred that the inhibited NEAT1 may repress liver fibrosis in ASH mice by regulating miR-129-5p and SOCS2.

\section{Materials and methods \\ Ethics statement}

Written informed consents were acquired from all patients before this study. The protocol of this study was confirmed by the Ethic Committee of First hospital of Jilin university. The protocol of animal experiments was approved by the Institutional Animal Care and Use Committee of First hospital of Jilin university.

\section{Study subjects}

Sixty-five cases of ASH patients (47 males and 18 females, aging 21-66 years, mean age of $39.82 \pm 6.07$ years) who were in line with the diagnostic criteria of ASH [21] and accepted treatment in First hospital of Jilin university from April 2017 to March 2018 were selected. Patients with diabetes, coronary heart disease, hyperlipidemia, hypertension, or other cardio-cerebral vascular diseases; malignant tumor, viral hepatitis, drug-induced hepatitis, or other non-alcoholic hepatic injury; women during pregnant stage or breast-feed stage, or patients with a poor compliance were excluded. Sixty-five people with healthy examination ( 41 males and 24 females, mean age of $(41.75 \pm 8.26$ years $)$ were also collected. Peripheral venous blood $(5 \mathrm{~mL})$ from ASH patients and people with healthy examination were preserved at $-4{ }^{\circ} \mathrm{C}$ for $30 \mathrm{~min}$ and centrifuged to collect serum, in which alanine aminotransferase (ALT), aspartate aminotransferase (AST), total cholesterol (TC) and triglyceride (TG) in serum were determined by an automatic biochemical analyzer and its original kit (Shenzhen Mindray Software Technology Co., Ltd., Shenzhen, China), as well as tumor necrosis factor- $\alpha$ (TNF- $\alpha$ ), interleukin-1 $\beta$ (IL-1 $\beta$ ) and IL-6 in serum by enzyme-linked immunosorbent assay (ELISA) kits (Beckman Coulter Life Sciences, Brea, CA, USA).

\section{Cell culture and treatment}

Mouse liver cell line AML-12 was purchased from Shanghai Institute of Biochemistry and Cell Biology, Chinese Academy of Sciences (Shanghai, China) and cultured in DMEM-F12 medium containing 10\% fetal bovine serum (FBS). Upon cell adherence, they were passaged the second day, and those in the logarithmic growth phase were used in the experiments.

AML-12 cells were stimulated by $100 \mathrm{mM}$ ethanol for $24 \mathrm{~h}$ alone, or stimulated by $100 \mathrm{mM}$ ethanol for $24 \mathrm{~h}$, followed by transfection of siRNA-NEAT1 negative control (NC), siRNA-NEAT1, miR-129-5p mimic NC, miR-129-5p mimic, NEAT1 overexpression vector (overexpressed (oe)-NEAT1) together with miR-129-5p mimic NC, or oe-NEAT1 together with miR-129-5p mimic. The siRNA-NC, siRNA-NEAT1, mimic-NC, miR-129-5p mimic and oe-NEAT1 were all acquired from GenePharma Co., Ltd, (Shanghai, China).

AML12 cells were seeded in 12-well plates and cultured for $24 \mathrm{~h}$ before transfection. The transfection was conducted by Lipofectamine 2000 reagent (Invitrogen Inc., Carlsbad, CA, USA) when the cell confluence reached 70-90\%. Transfected for $6 \mathrm{~h}$, the medium was changed and cells were stimulated by $100 \mathrm{mM}$ ethanol for $24 \mathrm{~h}$. 
Finally, cells and cell supernatant were collected for the subsequent cell experiments.

\section{3-(4,5-dimethyl-2-thiazolyl)-2,5-diphenyl-2-H-tetrazolium bromide (MTT) assay}

Cells were detached and made into single cell suspension, which was seeded onto 96-well plates at $100 \mu \mathrm{L} /$ well $\left(1.0 \times 10^{4}\right.$ cells/well $)$, and the marginal wells were appended with phosphate buffered saline (PBS). The adherent cells were stimulated by different concentrations of ethanol (6 duplicates were set) and cultured for $24 \mathrm{~h}$. Then, cells were supplemented with $20 \mu \mathrm{L}$ MTT solution $(5 \mathrm{mg} / \mathrm{mL})$, wrapped by silver paper and then cultured for $4 \mathrm{~h}$. After that, cells were added with $150 \mu \mathrm{L}$ dimethyl sulfoxide and cultured without light exposure for $10 \mathrm{~min}$. The optical density value was analyzed by a microplate reader.

\section{Experimental animals}

A total of 80 male C57BL/6 mice (aging $4 \mathrm{w}$, weighing $18-20 \mathrm{~g})$ that acquired from Laboratory Animal Center of Jilin University (Changchun, China) were adaptively fed at $20-25^{\circ} \mathrm{C}$ and $55 \%$ humidity in a clean environment for $1 \mathrm{w}$, with free access to food and water and normal $12 \mathrm{~h}$ day/night cycle.

\section{Establishment of ASH mice models}

The mice were fed with Lieber-DeCarli ad libitum diet (Dyets Inc. Bethlehem, PA, USA) and 5\% (vol/vol) ethanol (36\% ethanol-derived calories) for 4-7 w, and were given $20 \%$ ethanol ( $5 \mathrm{~g} / \mathrm{kg}$, body weight) by gavage. Mice for control $(n=10)$ were fed and given maltodextrin (same calories with the ethanol). The fodder was freshly configured every day and gavage was conducted in the last day of the modeling [22].

\section{Animal treatment}

The 70 successfully modeled mice were injected with lentiviruses expressing siRNA-NC, siRNA-NEAT1, agomiRNC, miR-129-5p agomiR, Oe-NEAT1+agomiR-NC, or Oe-NEAT1 + miR-129-5p agomiR (each $100 \mu \mathrm{L}$ ) [23] at caudal vein. The aforementioned lentivirus vector or agomir was constructed by GenePharma. After 4-w continuous injection, mice were euthanized to collect eyeball blood and liver tissues.

\section{Determination of serum factors}

The supernatant of AML-12 cells and mouse eyeball blood were collected, in which ALT and AST were determined by a fully automatic biochemical analyzer and its matching kits (both from Mindray Biomedical
Electronics Co., Ltd., Guangdong, China), TC and TG by microplate reader colorimetry (microplate reader was purchased from Shanghai Labsystems Biotechnology Co., Ltd., Shanghai, China) and TNF- $\alpha$, IL- $1 \beta$ and IL- 6 contents by ELISA kits (Beckman Coulter Life Sciences).

\section{Hematoxylin-eosin (HE) staining and oil red $\mathrm{O}$ staining}

HE staining: the mouse liver tissues were fixed, embedded, sectioned and stained. Liver tissues $(1 \mathrm{~cm} \times 1 \mathrm{~cm})$ were fixed with $4 \%$ paraformaldehyde for $24 \mathrm{~h}$, dehydrated by gradient ethanol, permeabilized by $1 / 2$ xylene and $1 / 2$ ethanol, embedded by paraffin and sectioned. The sections were stained by hematoxylin for $15 \mathrm{~min}$, differentiated by hydrochloric alcohol for $10 \mathrm{~s}$, washed by tap water for $1 \mathrm{~min}$, stained by $0.5 \%$ eosin for $3 \mathrm{~min}$, and then soaked in ethanol for $5 \mathrm{~min}$. After permeabilized and sealed, the sections were observed under a microscope.

Oil red O staining: mouse liver tissues were embedded, sectioned and stained. The pathology of liver tissues was observed under a light exposure. The sections $(10-\mu \mathrm{m})$ were dried for $15 \mathrm{~min}$, incubated by 100\% isopropanol for $5 \mathrm{~min}$, stained by $0.5 \%$ oil red $\mathrm{O}$ solution for $10 \mathrm{~min}$, toasted at $60^{\circ} \mathrm{C}$, and then rinsed by $85 \%$ isopropanol solution for $3 \mathrm{~min}$. After counterstained by hematoxylin for $2 \mathrm{~min}$, the sections were sealed by glycerin gelatin and observed under a microscope.

\section{Terminal deoxynucleotidyl transferase-mediated dUTP nick end-labeling (TUNEL) staining}

The cell apoptosis in liver tissue was determined by TUNEL staining (Beijing Zhongshu GoldenBridge Biotechnology Co., Ltd., Beijing, China). The sections were developed by diaminobenzidine and counterstained by hematoxylin, then the apoptotic cells were observed under a light microscope (Olympus Optical Co., Ltd, Tokyo, Japan). Apoptotic index $(\mathrm{AI})=$ the number of apoptotic cells/the number of total cells $\times 100 \%$.

\section{Masson's staining}

The tissues were normally sectioned, dewaxed, dehydrated and stained by Masson kits (Service Biological Technology, Wuhan, China). The collagen deposition was observed using a light microscope and the images were analyzed by the Image-Pro Plus software. 


\section{Reverse transcription quantitative polymerase chain reaction ( $R T-q P C R)$}

Trizol reagent (Life Technologies, Carlsbad, CA, USA) was employed to extract total RNA in serum, tissues and cells. RNA was reversely transcribed into cDNA via RTqPCR kits (Takara, Kusatsu, Shiga, Japan). PCR was conducted by SYBR Green PCR Master Mix kits (Roche, Palo Alto, CA, USA) and the data were analyzed by $2^{-\Delta \Delta C t}$ method [24]. U6 was taken as the loading control of miR129-5p and glyceraldehyde phosphate dehydrogenase (GAPDH) of NEAT1, SOCS2, collagen-I, and collagenIII. The primers (Table 1) were synthesized by Invitrogen.

\section{Western blot analysis}

Total proteins in serum, tissues and cells were evaluated by bicinchoninic acid kits (Pierce, Rockford, IL, USA). The extracted proteins that had been denatured by high temperature were mixed with loading buffer, boiled at $95{ }^{\circ} \mathrm{C}$ for $10 \mathrm{~min}$, conducted with $10 \%$ sodium dodecyl sulfate-polyacrylamide gel electrophoresis and transferred onto the polyvinylidene fluoride membranes. After fixed with 5\% bovine serum albumin (10-L16, Beijing Zhongshenglikang Technology Co., Ltd., Beijing, China), the membranes were appended with primary antibodies SOCS2 (1: 300, Abcam Inc., Cambridge, MA, USA) and GAPDH (1: 1000, Cell Signaling Technology, Beverly, MA, USA), and the horseradish peroxidase-conjugated secondary antibody (1: 5000; ab6721, Abcam). Next, the

\section{Table 1 Primer sequence}

\begin{tabular}{|c|c|}
\hline Gene & Sequence \\
\hline \multirow[t]{2}{*}{ NEAT1 } & F: 5'-CTCACTAAAGGCACCGAAG-3' \\
\hline & R: 5'-GGCAGAGAAGTTGCTTGTGG-3' \\
\hline \multirow[t]{2}{*}{ MiR-129-5p } & F: 5'-CACTTGGCTGCCCGATACTCT-3' \\
\hline & R: 5'-GCCCATGTTGTCCTGGATGTT-3' \\
\hline \multirow[t]{2}{*}{ SOCS2 } & F: 5'-AGCAGTTTGACAGCGTGGTT-3' \\
\hline & R: $5^{\prime}-C A G G T A A A G G T G A A C A G T C C C-3^{\prime}$ \\
\hline \multirow[t]{2}{*}{ Collagen-I } & F: 5'-GAGGGCCAAGACGAAGACATC-3' \\
\hline & R: $5^{\prime}-C A G A T C A C G T C A T C G C A C A A C-3^{\prime}$ \\
\hline \multirow[t]{2}{*}{ Collagen-III } & F: 5'-GGAGCTGGCTACTTCTCGC-3' \\
\hline & R: 5'-GGGAACATCCTCCTTCAACAG-3' \\
\hline \multirow[t]{2}{*}{ U6 } & F: 5'-GCTCGCTTCGGCAGCACA-3' \\
\hline & R: 5'-GAGGTATTCGCACCAGAGGA-3' \\
\hline \multirow[t]{2}{*}{ GAPDH } & F: 5'-ACGGCAAGTTCAACGGCACAG-3' \\
\hline & R: 5'-GACGCCAGTAGACTCCACGACA-3' \\
\hline
\end{tabular}

$F$ forward, $R$ reverse, NEAT1 nuclear paraspeckle assembly transcript 1, miR129-5p microRNA-129-5p, SOCS2 suppressor of cytokine signaling 2, GAPDH glyceraldehyde phosphate dehydrogenase

membranes were developed by enhanced chemiluminescent reagent and exposed in a dark room. GAPDH was taken as the internal reference and the data were analyzed by the Bio-Rad Image Lab system (GEL DOC EZ IMAGER, Bio-rad, CA, USA). Gray values of the protein bands were analyzed by the Image J software.

\section{Dual luciferase reporter gene assay}

The binding sites of NEAT1 and miR-129-5p, together with miR-129-5p and SOCS2 were predicted and analyzed by bioinformatic websites https://starbase.sysu. edu.cn/ and https://www.targetscan.org/vert_72/. The predicted binding sequences and their corresponding mutated sequence were inserted into pmirGLO vector (Promega, Madison, WI, USA) to build pmirGLONEAT1-wild type (Wt), pmirGLO-NEAT1-mutant (Mut), pmirGLO-SOCS2-Wt, and pmirGLO-SOCS2Mut. Meanwhile, the Wt and Mut vectors were co-transfected with mimic-NC or miR-129-5p mimic into $293 \mathrm{~T}$ cells for $24 \mathrm{~h}$. Luciferase activity was detected by DualLucy Assay Kit (Solarbio, Beijing, China).

\section{RNA-pull down assay}

NEAT1-Wt and NEAT1-Mut (50 nM) labeled by biotin were severally transfected into cells for $48 \mathrm{~h}$ and incubated by particular cell lysis buffer (Ambion Company, Austin, TX, USA) for $10 \mathrm{~min}$. Next, $50 \mathrm{~mL}$ cell lysate was sub-packaged and the rest lysate was co-cultured with M-280 streptavidin beads (Sigma-Aldrich, St Louis, MO, USA) coated with RNase-free and yeast tRNA (SigmaAldrich). Total RNA was extracted by Trizol and miR129-5p level was evaluated by RT-qPCR.

\section{Statistical analysis}

All data analyses were conducted using SPSS 21.0 software (IBM Corp. Armonk, NY, USA). The measurement data were expressed as mean \pm standard deviation, and Pearson correlation analysis was employed for correlation test. The unpaired $t$-test was performed for comparisons between two groups, one-way analysis of variance (ANOVA) for those among multiple groups and Tukey's post hoc test for pairwise comparisons. $P$ value $<0.05$ was indicative of statistically significant difference.

\section{Results}

NEAT1 and SOCS2 are highly expressed while miR-129-5p is poorly expressed in serum of ASH patients

The levels of corresponding indicators of ASH patients and healthy controls were evaluated (Table 2), and the results reflected that body mass index (BMI), ALT, AST, TC, TG, TNF- $\alpha$, IL- $1 \beta$ and IL- 6 levels were higher in ASH patients.

NEAT1, miR-129-5p and SOCS2 in serum of ASH patients and healthy controls were determined by RTqPCR and Western blot analysis. The outcomes indicated 
Table 2 Levels of corresponding indicators of ASH patients and healthy people

\begin{tabular}{lccl}
\hline Indicators & The ASH group $(\mathbf{n = 6 5})$ & $\begin{array}{l}\text { The healthy } \\
\text { group }(\mathbf{n}=65)\end{array}$ & $\boldsymbol{P}$ \\
\hline BMI $\left(\mathrm{Kg} / \mathrm{m}^{2}\right)$ & $28.57 \pm 5.26$ & $22.49 \pm 4.32$ & $<0.001$ \\
ALT $(\mathrm{U} / \mathrm{L})$ & $33.57 \pm 12.16$ & $19.75 \pm 10.21$ & $<0.001$ \\
AST $(\mathrm{U} / \mathrm{L})$ & $62.79 \pm 24.51$ & $26.14 \pm 13.87$ & $<0.001$ \\
TC $(\mathrm{mmol} / \mathrm{L})$ & $5.39 \pm 0.94$ & $4.02 \pm 0.76$ & $<0.001$ \\
TG $(\mathrm{mmol} / \mathrm{L})$ & $2.68 \pm 0.83$ & $1.47 \pm 0.66$ & $<0.001$ \\
TNF- $\mathrm{a}(\mathrm{pg} / \mathrm{mL})$ & $149.75 \pm 21.62$ & $25.68 \pm 6.03$ & $<0.001$ \\
IL-1 $\beta(\mathrm{pg} / \mathrm{mL})$ & $54.82 \pm 6.77$ & $23.25 \pm 2.41$ & $<0.001$ \\
IL-6 $(\mathrm{pg} / \mathrm{mL})$ & $211.35 \pm 49.08$ & $40.90 \pm 11.37$ & $<0.001$ \\
\hline
\end{tabular}

$A S H$ alcoholic steatohepatitis, $B M I$ body mass index, ALT alanine aminotransferase, AST aspartate aminotransferase, $T C$ total cholesterol, TG triglyceride, TNF- $\alpha$ tumor necrosis factor- $\alpha$, IL-1 $\beta$ interleukin- $1 \beta$, IL-6 interleukin- 6

that (Fig. 1a, b) NEAT1 and SOCS2 levels were elevated while miR-129-5p expression was inhibited in ASH patients.

The relations between NEAT1 and miR-129-5p, and between miR-129-5p and SOCS2 mRNA in serum of ASH patients were analyzed by Pearson correlation analysis (Fig. 1c, d), and the results mirrored that NEAT1 and miR-129-5p were in a negative relation $(r=-0.654$, $P<0.001)$, and miR-129 was also negatively related with SOCS2 mRNA $(\mathrm{r}=-0.618, P<0.001)$.
Inhibited NEAT1 or elevated miR-129-5p inhibits the elevated lipid metabolism and restrains inflammation reaction in ethanol-stimulated AML-12 cells

MTT assay observed the effect of different concentrations of ethanol on AML-12 cell viability, and the results (Fig. 2a) revealed that ethanol over $100 \mathrm{mM}$ concentration indeed impaired cell viability. Hence, $100 \mathrm{mM}$ ethanol was chosen in the subsequent experiments in vitro. NEAT1, miR-129-5p and SOCS2 expression in ethanoltreated AML-12 cells were determined by RT-qPCR and Western blot analysis and the outcomes (Fig. 2b) suggested that NEAT1 and SOCS2 expression were increased while miR-129-5p expression was reduced.

AML-12 cells were transfected with siRNAs, oligonucleotides or plasmids and stimulated by ethanol for $24 \mathrm{~h}$. It was demonstrated that NEAT1 and SOCS2 expression was decreased while miR-129-5p expression was increased by NEAT 1 down-regulation. SOCS 2 expression was reduced but miR-129-5p expression was advanced by miR-129-5p up-regulation. Up-regulating miR-129-5p reversed the effect of highly expressed NEAT1 on SOCS2 expression in AML-12 cells (Fig. 2c).

Measurement of lipid content and inflammatory factors mirrored that (Fig. 2d, e) down-regulating NEAT1 or upregulating miR-129-5p reduced TC, TG, TNF- $\alpha$, IL- $1 \beta$ and IL-6. Restoring miR-129-5p reversed the effect of overexpressed NEAT1 on lipid content and inflammatory factors in ethanol-stimulated AML-12 cells.
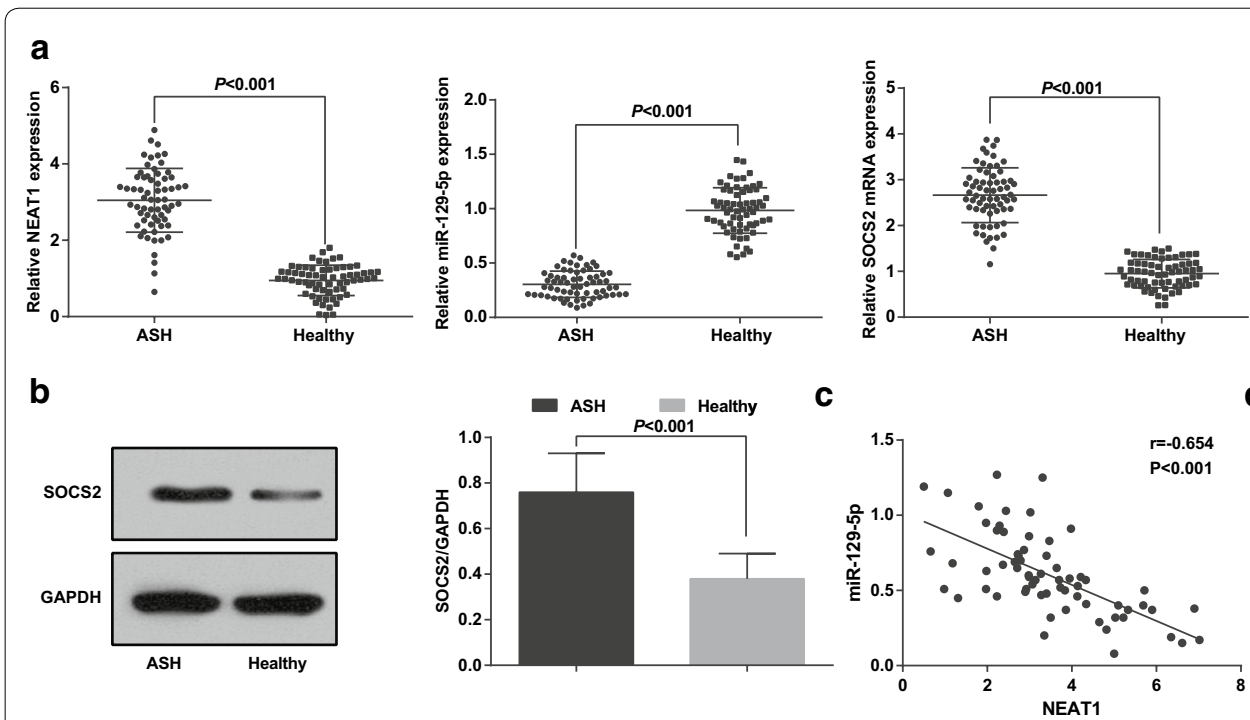

d

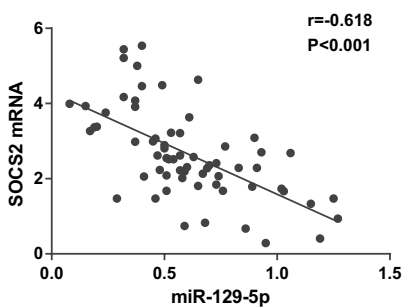

Fig. 1 LnCRNA NEAT1 and SOCS2 are highly expressed while miR-129-5p is poorly expressed in serum of ASH patients. A, expression of NEAT1, miR-129-5p, and SOCS2 in the ASH group and the healthy group; B, protein expression of SOCS2 in the ASH group and the healthy group; C, relation between the expression of miR-129-5p and NEAT1 in serum of ASH patients was analyzed by Pearson correlation analysis; $D$, relation between the expression of miR-129-5p and SOCS2 mRNA in serum of ASH patients was analyzed by Pearson correlation analysis; $n=65$, the measurement data were expressed as mean \pm standard deviation, and the unpaired t-test was performed for comparisons between two groups 


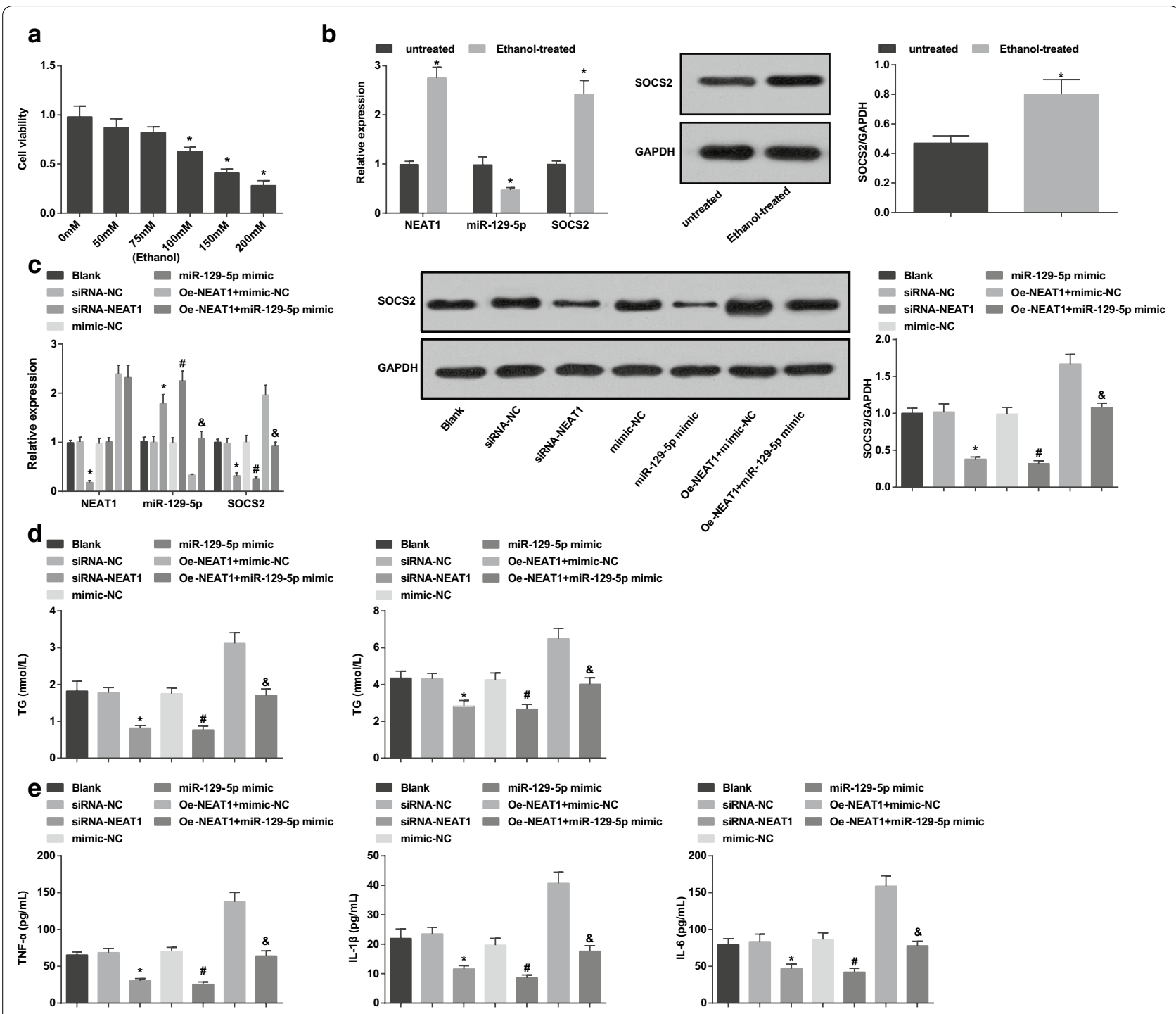

Fig. 2 Inhibited NEAT1 or elevated miR-129-5p inhibits the elevated lipid metabolism and restrains inflammation reaction in ethanol-stimulated AML-12 cells. A, impacts of different concentrations of ethanol on AML-12 cell viability were evaluated by MTT assay; B, the expression of NEAT1, miR-129-5p, and SOCS2 in AML-12 cells in the ethanol untreated group and the ethanol treated group; $C$, the expression of NEAT1, miR-129-5p, and SOCS2 in AML-12 cells that have been treated with ethanol after transfection; D, contents of TC and TG in AML-12 cells that have been treated with ethanol after transfection; E, contents of TNF- $a$, IL-1 $\beta$, and IL-6 in AML-12 cells that have been treated with ethanol after transfection; $\mathbf{a} * P<0.05$ vs the AML-12 cells that have not been stimulated by ethanol; $\mathbf{b}{ }^{*} P<0.05$ vs the untreated group; $\mathbf{c}-\mathbf{e}{ }^{*} P<0.05$ vs the siRNA-NC group, ${ }^{\#} P<0.05$ vs the mimic-NC group, ${ }^{\&} P<0.05$ vs the oe-NEAT1 + mimic-NC group; $N=3$, the measurement data were expressed as mean \pm standard deviation, one-way ANOVA was used for comparisons among multiple groups, and Tukey's post hoc test was used for pairwise comparisons after one-way ANOVA

Inhibited NEAT1 or elevated miR-129-5p promotes liver function and suppresses blood lipid and inflammation reaction in ASH mice
NEAT1, miR-129-5p and SOCS2 expression in liver tissues of ASH mice were measured and the outcomes (Fig. 3a) indicated that NEAT1 and SOCS2 expression 


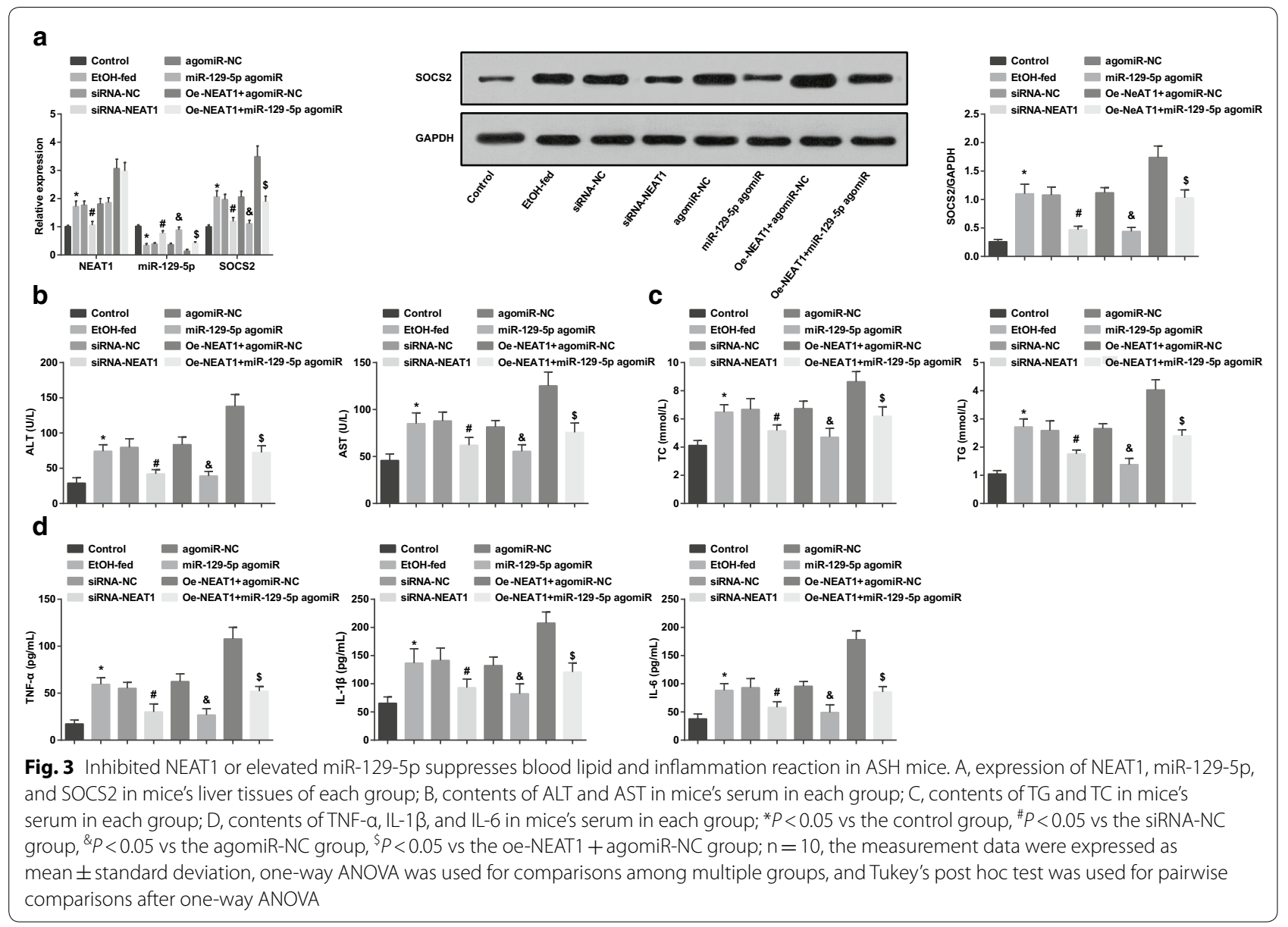

were elevated while miR-129-5p expression was repressed in ASH mice. Silencing NEAT1 restricted NEAT1 and SOCS2 expression and promoted miR129-5p expression. Enhancing miR-129-5p reduced SOCS2 expression but raised miR-129-5p expression. miR-129-5p elevation reversed the effect of overexpressed NEAT1 on SOCS2 expression in ASH mice.

The levels of serum lipid of mice were determined, and we have found that (Fig. 3b-d) TC, TG, TNF- $\alpha$, IL- $1 \beta$ and IL- 6 contents were increased in ASH mice, which were suppressed by knocking down NEAT1 or elevating miR-129-5p. miR-129-5p restoration reversed the effect of NEAT1 overexpression on blood lipid and inflammation reaction. These finding suggested that the silenced NEAT1 or up-regulated miR129-5p was able to promote the liver function and repress blood lipid and inflammation reaction in $\mathrm{ASH}$ mice.

\section{Inhibited NEAT1 or elevated miR-129-5p relieves pathology of liver tissues and apoptosis of hepatocytes in ASH mice}

The pathology of mouse liver tissue and lipid droplets were observed by $\mathrm{HE}$ staining and oil red $\mathrm{O}$ staining. The outcomes revealed that (Fig. 4a, b) the hepatocytes in liver tissues of normal mice were normal and orderly arranged, and there was no lipid vacuole or obvious lipid droplet. In ASH mice or AHS mice that were treated with lentiviruses expressing siRNA-NC, agomiR-NC or oe-NEAT1 + miR-129-5p agomiR, there were necrotic tissues, blurred liver lobule structure, abundant lipid vacuoles and inflammatory cell infiltration, as well as increased lipid droplets. In AHS mice that were treated with lentiviruses expressing siRNANEAT1 or miR-129-5p agomiR, the steatosis, inflammation reaction and cell necrosis were abated and lipid droplets were decreased. The pathological damage was aggravated and lipid droplets were further augmented in AHS mice that were treated with lentiviruses expressing oe-NEAT1 + agomiR-NC. 


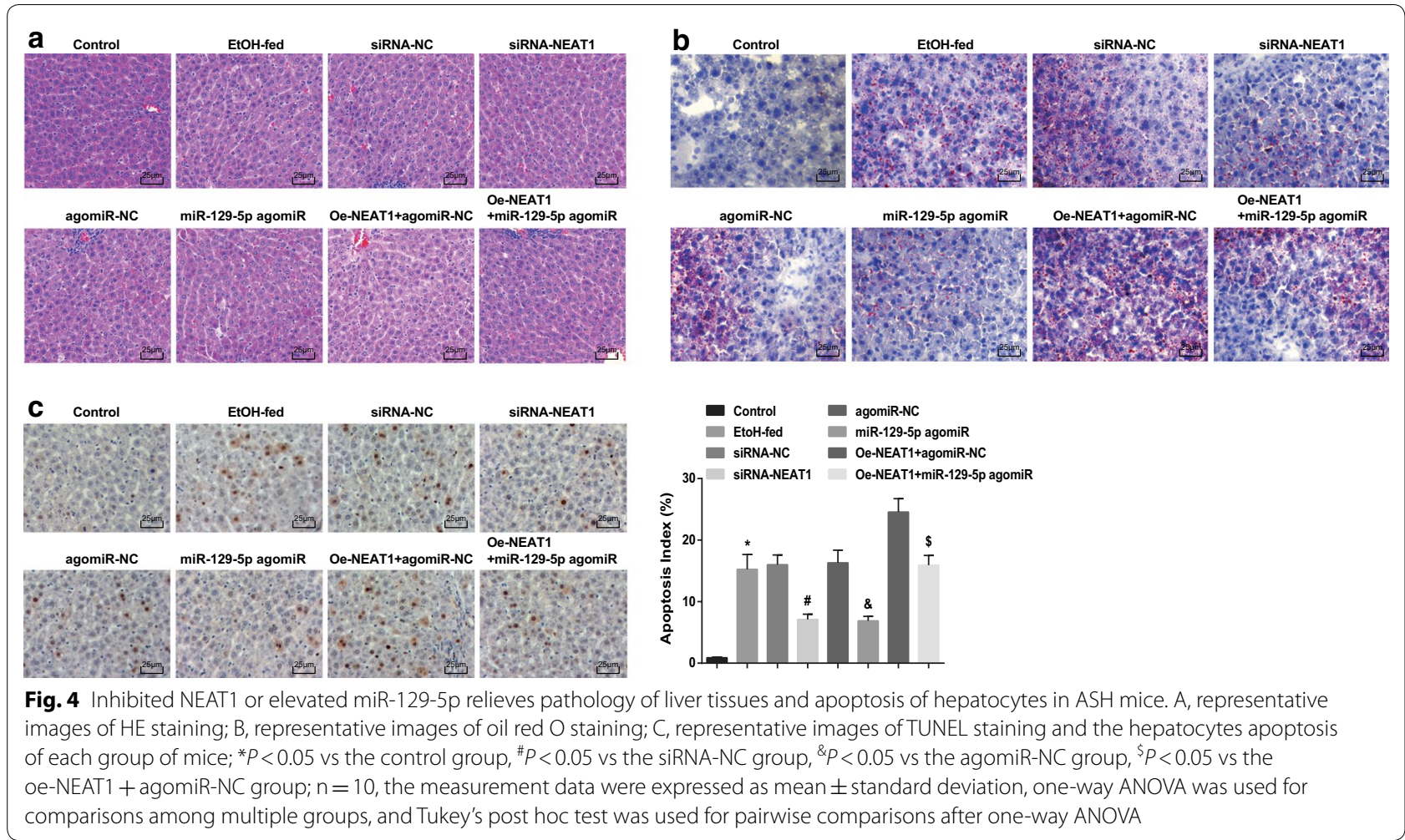

The apoptosis of hepatocytes in mouse liver tissue was observed by TUNEL staining. The nuclei of the apoptotic hepatocytes were stained into brown and we have found that (Fig. 4c) ASH mice showed increased AI, which were reduced by down-regulated NEAT1 or up-regulated miR129-5p. miR-129-5p enhancement attenuated the effect of NEAT1 overexpression on AI of hepatocytes. It was hinted that inhibited NEAT1 or elevated miR-129-5p could relieve pathology of liver tissues and apoptosis of hepatocytes in ASH mice.

\section{Inhibited NEAT1 or elevated miR-129-5p ameliorates liver fibrosis in ASH mice}

Masson's staining was employed to observe the fibrous hyperplasia of mouse liver tissues and the outcomes (Fig. 5a) suggested that there were plump and orderly arranged hepatocytes, clear liver lobule structure, infinitesimal blue in central vein and blood vessel wall of portal area, and no obvious collagen fiber hyperplasia in liver tissues of the normal mice. In ASH mice or AHS mice that were treated with lentiviruses expressing siRNA-NC, agomiR-NC or oe-NEAT1 + miR-129-5p agomiR, there were abundant blue adherence in portal area, increased and broadened fibrous septum connected into bridges, fake lobule formed by fibrous septum, and obvious collagen fiber hyperplasia in mouse liver tissues. The collagen fiber hyperplasia and hyperplasia of connective tissues around the central vein and portal area were reduced in liver tissues of AHS mice that were treated with lentiviruses expressing siRNA-NEAT1 or miR-129-5p agomiR while increased in AHS mice that were treated with lentiviruses expressing oe-NEAT1 + agomiR-NC.

The levels of fibrous factors in mouse liver tissue presented that (Fig. 5b, c) collagen-I and collagen-III expression were enhanced in ASH mice, which would be attenuated by suppressing NEAT1 or increasing miR129-5p. Up-regulating miR-129-5p reversed the effect of NEAT1 overexpression on fibrosis-related factors in liver tissues of ASH mice.

\section{MiR-129-5p particularly binds with NEAT1, and SOCS2 is targeted by miR-129-5p}

According to the bioinformatic website (https://starbase. sysu.edu.cn/) (Fig. 6a), we have discovered that NEAT1 could bind with miR-129-5p. As confirmed by dual luciferase reporter gene assay (Fig. 6b), miR-129-5p overexpression markedly suppressed luciferase expression driven by pmirGLO-NEAT1-Wt but not that driven by pmirGLO-NEAT1-Mut, suggesting that miR-129-5p could bind with NEAT1. RNA pull-down assay further testified that bio-NEAT1-Wt could enrich more miR-129-5p in contrast to the bio-NEAT1-Mut and bio-probe-NC (Fig. 6c). We could conclude based on 


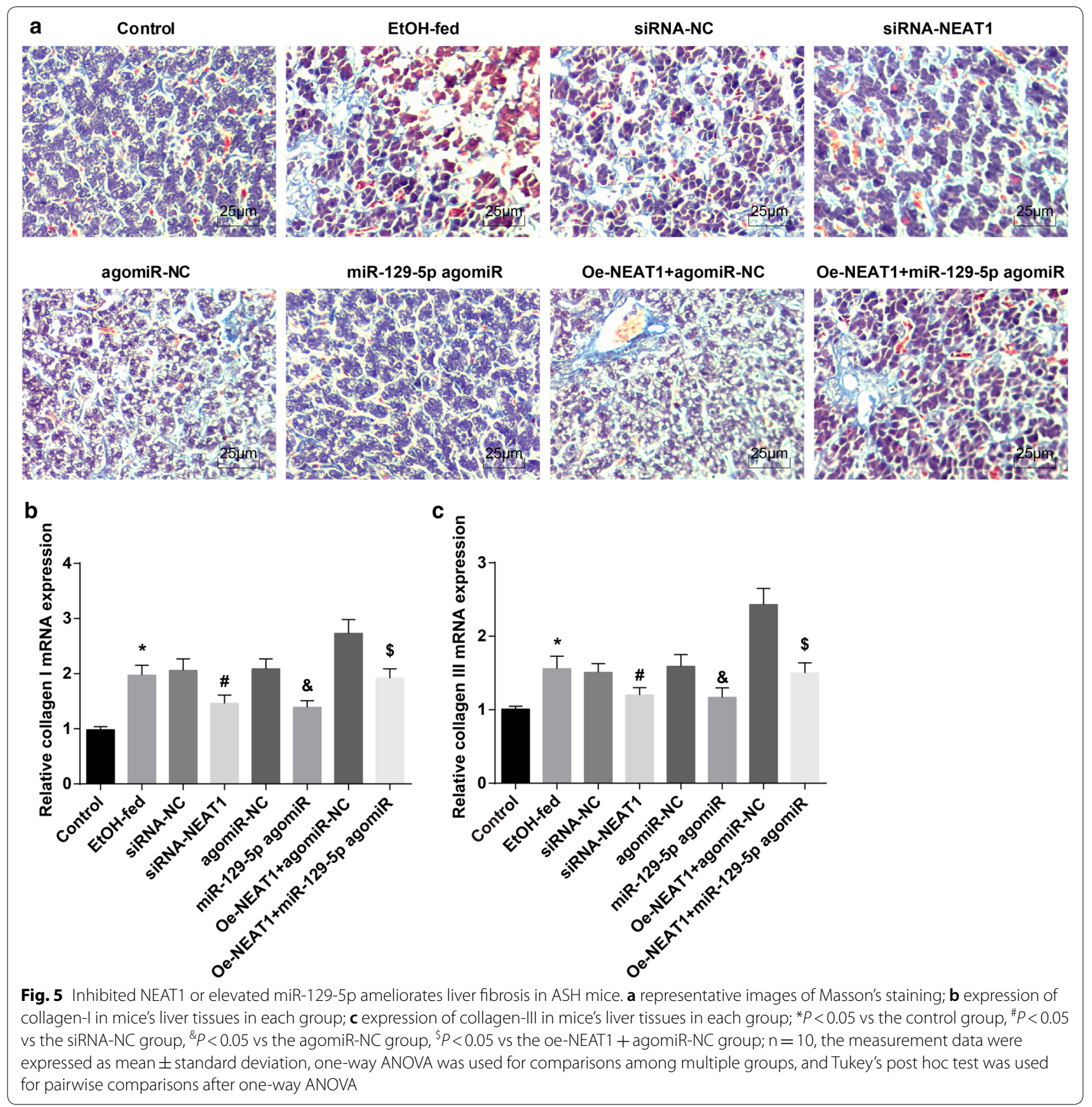

these results that NEAT1 served as a ceRNA to absorb miR-129-5p.

As predicted by the bioinformatic website (https:// www.targetscan.org/vert_72/) (Fig. 6d), miR-129-5p could bind with SOCS2. The target relation between miR-129-5p and SOCS2 was confirmed by dual luciferase reporter gene assay (Fig. 6e), and the outcomes mirrored that the reporter with pmirGLO-SOCS2 3'-UTR-Wt exhibited weakened luciferase activity following cotransfection with miR-129-5p mimic. Nevertheless, the luciferase activity of the mutant reporter did not alter following miR-129-5p mimic transfection, indicating that SOCS2 was the direct target gene of miR-129-5p.

\section{Discussion}

Belonging to the ALDs, ASH may develop into liver fibrosis, cirrhosis, liver failure, or even $\mathrm{HCC}$, and it has been proved that the six-month mortality rate of severe ASH is $40 \%$ [3]. When it comes to the functional mechanisms of lncRNAs, the ceRNA hypothesis assumed that 


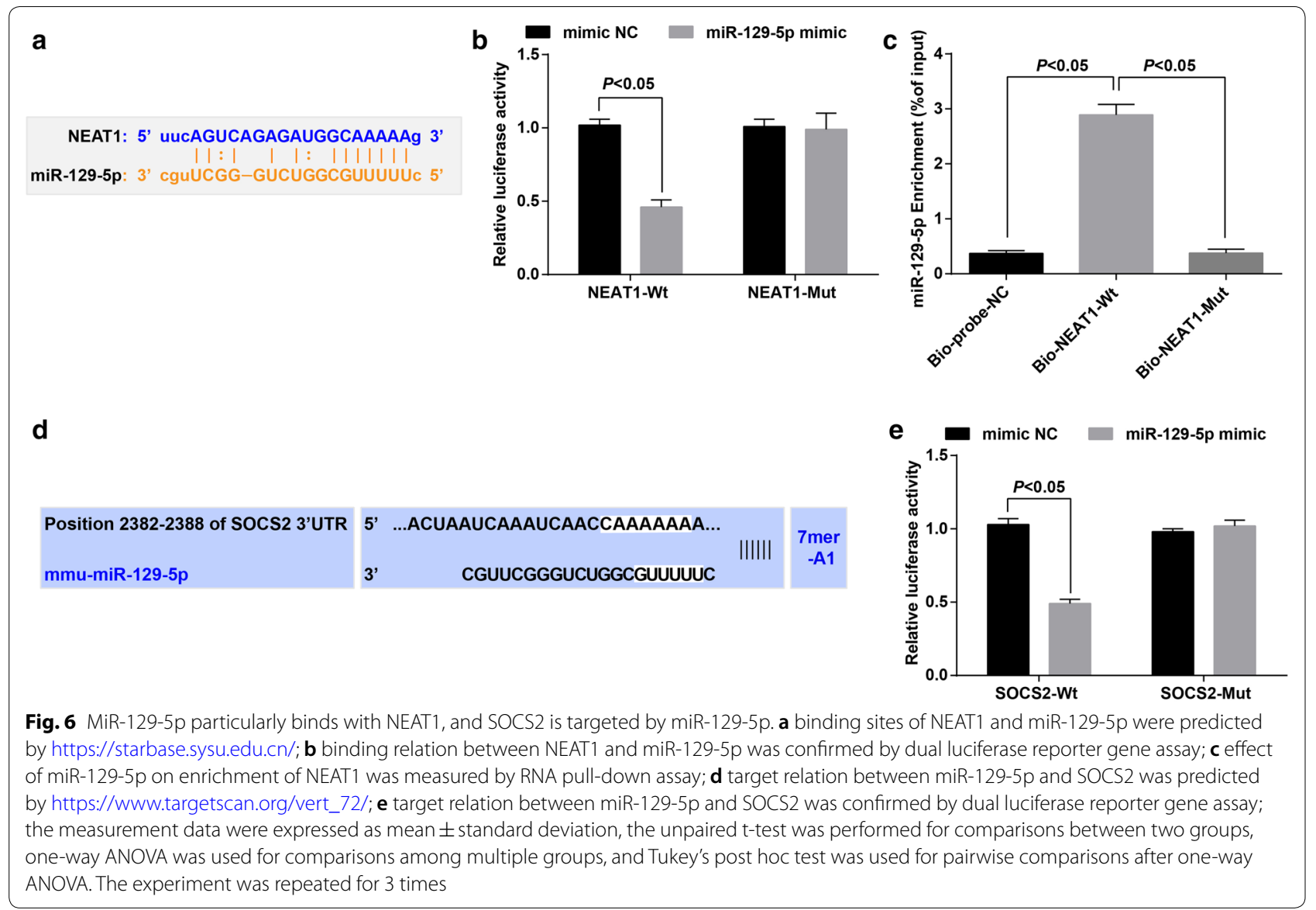

particular RNAs could impair the activity of miRNAs via sequestration, thus up-regulating their target genes [25]. Our research aimed to assess the role of the NEAT1/ miR-129-5p/SOCS2 axis in ASH development, and we have discovered that the inhibited NEAT1 could repress liver fibrosis and development of ASH through the elevation of miR-129-5p and suppression of SOCS2.

Several results have been concluded in this study and one of them suggested that NEAT1 and SOCS2 were highly expressed while miR-129-5p was poorly expressed in serum of ASH patients and mice models. Similarly, Chen et al. have figured out that NEAT1 was considerably up-regulated in NAFLD models [9], and a same tendency of NEAT1 in hepatocytes under NAFLD condition has also been demonstrated [10]. Evidences have suggested that the miR-129-5p expression was restrained in HCC tissues or cells $[13,14]$. As for the expression of SOCS2, a published literature has unveiled that the level of SOCS2 was elevated in rats that fed with Western style high-fat and high cholesterol diet, suggesting that SOCS2 was highly expressed in NAFLD rat models [19]. Moreover, we have found that NEAT1 could function as a ceRNA to sponge miR-129-5p, and the similar relation between NEAT1 and miR-129-5p has been elucidated in other researches as well $[15,16]$. Bioinformatic method and dual luciferase reporter gene assay in our study indicated a target relation between miR-129-5p and SOCS2. However, this relationship remains uncovered.

Another result in our research indicated that knockdown of NEAT1 was able to improve the lipid metabolism by amplifying miR-125-9p and inhibiting SOCS2 in ethanol-stimulated AML-12 cells. In consistent with this outcome, Liu et al. have illuminated that the degradation of NEAT1 had the capacity to reduce the levels of diacylglycerol and free fatty acid in rat HCC models, thereby modulating the abnormal lipid metabolism in hepatocytes [26], and Zadjali et al. have pointed out that the inhibition of SOCS2 could protect against hepatic steatosis in mice that fed with high-fat-diet [20]. In addition, we have unraveled that the down-regulation of NEAT1 and up-regulation of miR-129-5p were able to restrict the inflammation reaction in ASH mice by reducing SOCS2. In accordance with this outcome, Wang et al. have figured out that the suppression of NEAT1 could attenuate inflammation response and lipid uptake in human macrophages [27], and it has been clarified by a recent 
document that the elevation of miR-129-5p relieved neuroinflammation after ischemia-reperfusion [28]. Furthermore, we have discovered that the reduced NEAT1 or amplified miR-129-5p could decelerate the apoptosis of hepatocytes in ASH mice by targeting SOCS2. In line with the result, a recent study has reported that the repression of NEAT1 inhibited the apoptosis of human kidney tubular cells in acute kidney injury [29], it has also been illustrated by Zhang et al. that miR-129-5p overexpression had the ability to inhibit apoptosis of cardiomyocytes in ischemic heart disease [30], and Xue et al. have discovered that the reduction of SOCS2 protected cardiomyocytes from apoptosis that induced by myocardial ischemia/reperfusion injury [31]. Additionally, we have evidenced that the suppressed NEAT1 and SOCS2, and the promoted miR-129-5p were able to attenuate the liver fibrosis in ASH mice. A similar finding has been noted by $\mathrm{Yu}$ et al. that the deletion of NEAT1 restrained liver fibrosis in vivo and in vitro [32], and it has been revealed by a recent research that the inhibition of miR-129-5p contributed to the collagen I synthesis in hepatic stellate cells, which is the pathogenesis of liver fibrosis [33].

\section{Conclusion}

In conclusion, we have illustrated that the repression of NEAT1 and SOCS2, and upregulation of miR-129-5p could decelerate the development of ASH, which may provide novel evidence for the functional mechanisms of NEAT1/miR-129-5p/SOCS2 axis in ASH. We identified NEAT1 could play a regulatory role in liver function and provide novel insights into the modulation of hepatocyte viability in ASH. Moreover, this research also supplies the possibility of overcoming alcohol over-consumptionrelated diseases to some extent. Nevertheless, further studies are demanded to validate our results.

\section{Acknowledgement}

We would like to acknowledge the reviewers for their helpful comments on this paper.

\section{Authors' contributions \\ DS contributed to study design; JY contributed to manuscript editing; YL con- tributed to experimental studies; YY contributed to data analysis. All authors read and approved the final manuscript.}

\section{Funding}

This work was supported by Wu Jieping Medical Foundation (The role of KLF2 in the development of hepatocellular carcinoma and in the Regulation of Hedgehog signal pathway), Grant Number: 320.6750.17217.

\section{Availability of data and materials Not applicable.}

\section{Ethics approval and consent to participate}

Written informed consents were acquired from all patients before this study. The protocol of this study was confirmed by the Ethic Committee of First hospital of Jilin university. The protocol of animal experiments was approved by the Institutional Animal Care and Use Committee of First hospital of Jilin university.

\section{Consent for publication}

Not applicable.

\section{Competing interests}

The authors declare that they have no conflicts of interest.

\section{Author details}

${ }^{1}$ Department of Hepato-Biliary-Pancreatic Surgery, First Hospital, Jilin University, Changchun 130021, Jilin, People's Republic of China. ${ }^{2}$ Department of Ultrasonography, China-Japan Union Hospital, Jilin University, Changchun 130021, Jilin, People's Republic of China. ${ }^{3}$ Department of Colorectal \& Anal Surgery, First Hospital, Jilin University, No. 71 Xinmin street, Changchun 130021, Jilin, People's Republic of China.

Received: 23 March 2020 Accepted: 24 October 2020

Published online: 24 November 2020

\section{References}

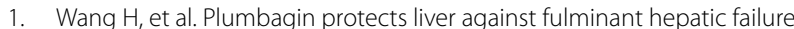
and chronic liver fibrosis via inhibiting inflammation and collagen production. Oncotarget. 2016;7(50):82864-75.

2. Petrasek J, et al. Differences in innate immune signaling between alcoholic and non-alcoholic steatohepatitis. J Gastroenterol Hepatol. 2013;28(Suppl 1):93-8.

3. Li TH, et al. Elafibranor interrupts adipose dysfunction-mediated gut and liver injury in mice with alcoholic steatohepatitis. Clin Sci. 2019;133(3):531-44.

4. Rehm J, Samokhvalov AV, Shield KD. Global burden of alcoholic liver diseases. J Hepatol. 2013;59(1):160-8.

5. Spahr L, et al. Early liver biopsy, intraparenchymal cholestasis, and prognosis in patients with alcoholic steatohepatitis. BMC Gastroenterol. 2011;11:115.

6. Lao Y, et al. Long noncoding RNA ENST00000455974 plays an oncogenic role through up-regulating JAG2 in human DNA mismatch repair-proficient colon cancer. Biochem Biophys Res Commun. 2019;508(2):339-47.

7. Ding F, et al. NEAT1/miR-23a-3p/KLF3: a novel regulatory axis in melanoma cancer progression. Cancer Cell Int. 2019;19:217.

8. Zhang CC, Niu F. LncRNA NEAT1 promotes inflammatory response in sepsis-induced liver injury via the Let-7a/TLR4 axis. Int Immunopharmacol. 2019;75:105731

9. Chen $X$, et al. LncRNA NEAT1 promotes hepatic lipid accumulation via regulating miR-146a-5p/ROCK1 in nonalcoholic fatty liver disease. Life Sci. 2019;235:116829

10. Sun Y, et al. LncRNA NEAT1-MicroRNA-140 axis exacerbates nonalcoholic fatty liver through interrupting AMPK/SREBP-1 signaling. Biochem Biophys Res Commun. 2019;516(2):584-90.

11. Yang D, et al. Inhibitory effect of MiR-449b on cancer cell growth and invasion through LGR4 in non-small-cell lung carcinoma. Curr Med Sci. 2018;38(4):582-9.

12. Chen YP, et al. Circulating MicroRNAs as potential biomarkers for alcoholic steatohepatitis. Liver Int. 2013;33(8):1257-65

13. Zhang $D$, et al. Long noncoding RNA PCAT-1 promotes invasion and metastasis via the miR-129-5p-HMGB1 signaling pathway in hepatocellular carcinoma. Biomed Pharmacother. 2017;95:1187-93.

14. Liu Y, et al. VCP/p97, down-regulated by microRNA-129-5p, could regulate the progression of hepatocellular carcinoma. PLoS ONE. 2012;7(4):e35800.

15. Fang $L$, et al. Long non-coding RNA NEAT1 promotes hepatocellular carcinoma cell proliferation through the regulation of miR-129-5p-VCPIkappaB. Am J Physiol Gastrointest Liver Physiol. 2017;313(2):G150-6.

16. Fu MC, et al. Nuclear paraspeckle assembly transcript 1 promotes the metastasis and epithelial-mesenchymal transition of hepatoblastoma cells by inhibiting miR-129-5p. Oncol Lett. 2017;14(5):5773-8.

17. Croker BA, Kiu H, Nicholson SE. SOCS regulation of the JAK/STAT signalling pathway. Semin Cell Dev Biol. 2008;19(4):414-22. 
18. Monti-Rocha R, et al. SOCS2 is critical for the balancing of immune response and oxidate stress protecting against acetaminophen-induced acute liver injury. Front Immunol. 2018;9:3134.

19. Yuan F, et al. Fish oil alleviated high-fat diet-induced non-alcoholic fatty liver disease via regulating hepatic lipids metabolism and metaflammation: a transcriptomic study. Lipids Health Dis. 2016;15:20

20. Zadjali F, et al. SOCS2 deletion protects against hepatic steatosis but worsens insulin resistance in high-fat-diet-fed mice. FASEB J. 2012;26(8):3282-91.

21. Takada A. Diagnostic criteria for alcoholic liver disease. Int Hepatol Commun. 1995;3(2):63-9.

22. Petrasek J, et al. IL-1 receptor antagonist ameliorates inflammasome-dependent alcoholic steatohepatitis in mice. J Clin Invest. 2012;122(10):3476-89.

23. Ding J, et al. Effect of miR-34a in regulating steatosis by targeting PPARalpha expression in nonalcoholic fatty liver disease. Sci Rep. 2015;5:13729.

24. Chen C, et al. Real-time quantification of microRNAs by stem-loop RTPCR. Nucleic Acids Res. 2005;33(20):e179.

25. Wang $X$, et al. LncRNA SNHG6 promotes chemoresistance through ULK1induced autophagy by sponging miR-26a-5p in colorectal cancer cells. Cancer Cell Int. 2019a;19:234.

26. Liu X, et al. Long non-coding RNA NEAT1-modulated abnormal lipolysis via ATGL drives hepatocellular carcinoma proliferation. Mol Cancer. 2018;17(1):90

27. Wang $L$, et al. Blockade of NEAT1 represses inflammation response and lipid uptake via modulating miR-342-3p in human macrophages THP-1 cells. J Cell Physiol. 2019b;234(4):5319-26.
28. LiXQ et al. Elevated microRNA-129-5p level ameliorates neuroinflammation and blood-spinal cord barrier damage after ischemia-reperfusion by inhibiting HMGB1 and the TLR3-cytokine pathway. J Neuroinflammation. 2017;14(1):205

29. Jiang $X$, et al. LncRNA NEAT1 promotes hypoxia-induced renal tubular epithelial apoptosis through downregulating miR-27a-3p. J Cell Biochem. 2019;120(9):16273-82.

30. Zhang H, Zhang X, Zhang J. MiR-129-5p inhibits autophagy and apoptosis of $\mathrm{H} 9 \mathrm{c} 2$ cells induced by hydrogen peroxide via the PI3K/AKT/mTOR signaling pathway by targeting ATG14. Biochem Biophys Res Commun. 2018;506(1):272-7.

31. Xue X, Luo L. LncRNA HIF1A-AS1 contributes to ventricular remodeling after myocardial ischemia/reperfusion injury by adsorption of microRNA-204 to regulating SOCS2 expression. Cell Cycle. 2019;18(19):2465-80.

32. Yu F, et al. NEAT1 accelerates the progression of liver fibrosis via regulation of microRNA-122 and Kruppel-like factor 6. J Mol Med (Berl). 2017;95(11):1191-202.

33. Chen $Y$, et al. Osteopontin promotes collagen I synthesis in hepatic stellate cells by miRNA-129-5p inhibition. Exp Cell Res. 2018;362(2):343-8.

\section{Publisher's Note}

Springer Nature remains neutral with regard to jurisdictional claims in published maps and institutional affiliations.
Ready to submit your research? Choose BMC and benefit from:

- fast, convenient online submission

- thorough peer review by experienced researchers in your field

- rapid publication on acceptance

- support for research data, including large and complex data types

- gold Open Access which fosters wider collaboration and increased citations

- maximum visibility for your research: over $100 \mathrm{M}$ website views per year

At $\mathrm{BMC}$, research is always in progress.

Learn more biomedcentral.com/submissions 